\title{
Elevated preoperative neutrophil/lymphocyte ratio as a predictor of increased long-term survival in minimal invasive coronary artery bypass surgery compared to sternotomy
}

\author{
Basem Azab ${ }^{1 *}$, Masood A Shariff², Rana Bachir², John P Nabagiez ${ }^{2}$ and Joseph T McGinn Jr,2
}

\begin{abstract}
Background: Neutrophil lymphocyte ratio (NLR) is a predictor of major adverse cardiovascular outcomes. Our study explores the value of NLR in predicting long-term mortality after minimally invasive coronary artery bypass surgery (MICS) via lateral left-thoracotomy versus conventional sternotomy coronary artery bypass grafting (CABG) surgery.

Methods: A total of 1126 consecutive patients (729 sternotomy CABG and 397 MICS) from a single tertiary center between 2005 and 2008 were followed until 2011. We stratified the patients into equal tertiles according to preoperative NLR. The primary outcome, all-cause mortality, was compared among the NLR tertiles.

Results: Out of the 1126 patients included in the study, 1030 (91\%) patients underwent off-pump CABG. The first (NLR <2.3) tertile had a significantly lower 5-year mortality $(30 / 371=8 \%)$ in comparison to the second (NLR $=2.3-3.4)$ and third (NLR $\geq 3.5)$ tertiles $(49 / 375=13 \%$ and $75 / 380=20 \%$ ), respectively with $p<0.0001$. After multivariate adjustment, NLR was a significant independent predictor of mortality (hazard ratio [HR] per each unit increase of NLR was 1.05, 95\% confidence interval [CI] 1.01-1.10, $p=0.008)$. MICS and sternotomy CABG groups with NLR $<3$ had similar mortality $(21 / 221=9.5 \%$ and $40 / 403=9.9 \%), p=1$. However among patients with NLR $\geq 3$, MICS had a significantly lower mortality $(23 / 176=13.1 \%)$ compared to the sternotomy CABG $(70 / 326=21.5 \%), p=0.02$.

According to the multivariate analysis of patients with NLR $\geq 3$, MICS had a significantly lower mortality compared to sternotomy CABG (HR=0.44, 95\% Cl 0.24-0.78, $p=0.005)$.

Conclusion: Elevated preoperative NLR is an independent predictor of long-term mortality after CABG. Among the patients with NLR $\geq 3$, MICS was associated with a significantly improved survival compared with sternotomy CABG.
\end{abstract}

Keywords: Inflammatory cells, Minimally invasive surgery, CABG, Thoracotomy, Outcomes

\section{Background}

Neutrophil lymphocyte ratio (NLR), a simple inflammatory marker, was found to be a significant independent predictor of adverse outcomes in patients with coronary artery disease [1-3]. The preoperative NLR was also a significant predictor of mortality among patients who underwent coronary artery bypass grafting surgery (CABG) [4]. Our goal was to evaluate the preoperative NLR as a predictor of mortality using our CABG population which includes both

\footnotetext{
* Correspondence: basemnady2000@yahoo.com

${ }^{1}$ Department of Surgery, Staten Island University Hospital, 475 Seaview Ave, Staten Island, NY, USA

Full list of author information is available at the end of the article
}

a conventional sternotomy CABG and the minimally invasive coronary artery bypass grafting (MICS) approach via left-thoracotomy [5]. We hypothesize that patients with elevated NLR, reflecting a higher preoperative inflammatory burden, will have better survival with MICS in comparison to sternotomy CABG. We evaluated the impact of these two different surgical approaches in patients with elevated NLR.

\section{Methods}

This is an observational study of a prospectively maintained database to explore the predictive value of NLR on longterm survival of 1,177 patients who underwent coronary 
bypass surgery at the Heart Institute of Staten Island University Hospital in Staten Island, New York, a population of 443,728, between January 2005 and December 2008. A total of 1126 patients were included in the study and followed through December 2010; mean follow-up was $49 \pm$ 15.2 months, with a range of 21 to 70 months. The only inclusion criterion was a preoperative complete blood count. Exclusion criteria included: hematological proliferative diseases, active or chronic inflammatory or autoimmune diseases, steroid therapy, concomitant valve surgery or unavailable complete blood count with differential preoperatively. Fifty-one patients were excluded from the study: 22 patients with concomitant valve surgery, 6 patients with hematological disease, 14 on steroids and 9 who did not have complete blood count with differential preoperatively. The primary end point, all cause five year mortality, was obtained from the electronic medical record and the social security death index. Differential leukocyte counts were obtained by the Coulter counter technique (Coulter Gen S Hematology Analyzer, Beckman Coulter Corp ${ }^{\bullet}$, Hialeah, Florida).

Trained physicians reviewed electronic medical records for potential confounders, including demographic variables, coronary artery disease risk factors, comorbidities, prescribed medications before surgery and medications at discharge, operative variables, laboratory values, left ventricular ejection fraction, New York Heart Association (NYHA) and Canadian Cardiovascular Society (CCS) class (Tables 1 and 2). We obtained left ventricular ejection fraction from echocardiography reports. Positive smoking status included both current and prior smokers. To study the baseline characteristics according to the NLR, the patients were divided into equal tertiles according to NLR: $1^{\text {st }}$ tertile (NLR $<2.2$ with 371 patients), $2^{\text {nd }}$ tertile $(2.2 \leq \mathrm{NLR} \leq 3.4$ with 375 patients), and $3^{\text {rd }}$ tertile (NLR $>3.4$ with 380 patients). The study was powered to evaluate the primary outcome with an estimated 7\% 5-year mortality (7\% versus 14\%) difference between patients in the $1^{\text {st }}$ and $3^{\text {rd }}$ NLR tertiles. To achieve this difference with an $80 \%$ power and a $5 \%$ type 1 error required a minimum of 305 patients in each NLR tertile.

The NLR tertiles were analyzed using the chi-square test for categorical variables and Kruskall-Wallis test for continuous variables. The distributions of continuous and categorical variables were represented as mean \pm standard deviation, frequencies and percentages, respectively. A univariate screen of all potential predictors of mortality using a separate Cox proportional hazards model for each variable was performed. Those variables that were found to be statistically significant $(\mathrm{p}<0.05)$ as well as clinically meaningful in the univariate analyses were included in a multivariate Cox regression model. Backwards selection with significance level for removal from the model set at 0.1 was used to remove variables which did not significantly contribute information to this model, given other factors included in the model.

In order to avoid collinearity preoperative neutrophils and lymphocytes were not included in the model with preoperative NLR. The analysis of mortality was accomplished by applying standard methods of survival analysis (i.e., computing the Kaplan-Meier product limit curves where the data was stratified by NLR tertiles). In cases where the endpoint event (death) had not yet occurred the number of months until last follow-up was used and considered censored. The three NLR groups were compared using the log-rank test. Additionally, among elevated NLR group, a multivariate logistic regression was performed to find the best model that fits the data and that explains the association between mortality and several independent variables deemed either to have significant association with mortality as a binary response or to be considered as clinically important. A backward selection procedure, with significance level for removal from the model set at 0.1 was used. The odds ratios (OR) and 95\% confidence interval (CI) of the best fitted model were computed. Two models were conducted in this multivariate stage. All analyses were performed using SPSS version 19.0 (SPSS Inc., Chicago, IL, USA). This study was approved by the institutional review board at Staten Island University Hospital.

\section{Results}

Out of the 1126 patients included in the study, 1030 (91\%) patients underwent off-pump CABG (MICS, 376/ $397=95 \%$; Sternotomy CABG, 654/729=90\%). The patients in the highest NLR tertile were significantly older with more Caucasian individuals in comparison to the lowest NLR tertile $(\mathrm{p}<0.0001)$. Additionally, the patients in the highest NLR tertile had significantly higher preoperative serum creatinine and glucose levels with a higher prevalence of diabetes, preoperative end stage renal disease requiring hemodialysis and family history of coronary artery disease compared to those in the lowest NLR tertile. The three NLR tertiles had similar distribution of both preoperative NYHA and CCS classes. Postoperatively, the patients in the highest NLR tertiles suffered a significantly higher rate of renal failure requiring hemodialysis and new onset atrial fibrillation in comparison to those in the lowest NLR tertile.

The patients in the lowest NLR tertile had significantly lower 30-day, 6-month, 1-year and 5-year mortality rates $(0.5 \%, 1.6 \%, 2.4 \%$ and $8 \%)$ in comparison to the middle NLR tertile $(1.3 \%, 4.3 \%, 5.1 \%$ and $13 \%)$ and the highest NLR tertile (2.7\%, 7.7\%, 9.9\% and 20\%), respectively with $\mathrm{p}<0.0001$. When we compared the overall survival among MICS and sternotomy subgroups in each NLR tertile, the MICS had significantly lower mortality only among the highest NLR tertile ( $p$ value $<0.001$ according to two tailed 
Table 1 Baseline characteristics (demographic and presentation) of coronary artery bypass patients according to neutrophil lymphocyte ratios (NLR) and surgical approach (MICS vs. Sternotomy CABG)

\begin{tabular}{|c|c|c|c|c|c|c|c|c|c|}
\hline \multirow[t]{2}{*}{ Variables } & \multicolumn{2}{|c|}{ NLR <2.3 (N = 371) } & \multirow[t]{2}{*}{$p$ Value } & \multicolumn{2}{|c|}{$2.3 \leq \mathrm{NLR} \leq 3.4(\mathrm{~N}=375)$} & \multirow[t]{2}{*}{$p$ Value } & \multicolumn{2}{|c|}{ NLR >3.4 (N = 380) } & \multirow[t]{2}{*}{$p$ Value } \\
\hline & Sternotomy CABG & MICS & & Sternotomy CABG & MICS & & Sternotomy CABG & MICS & \\
\hline n & 242 & 129 & & 245 & 130 & & 243 & 137 & \\
\hline Age (years) & $62.5 \pm 10.2$ & $60.7 \pm 10.9$ & 0.12 & $64.3 \pm 11.0$ & $62.6 \pm 10.7$ & 0.14 & $67.5 \pm 11.0$ & $65.6 \pm 10.7$ & 0.10 \\
\hline Male & $174(71.9 \%)$ & $90(69.8 \%)$ & 0.67 & $172(70.5 \%)$ & $93(71.0 \%)$ & 0.92 & $178(73.3 \%)$ & $100(73.0 \%)$ & 0.96 \\
\hline Race (Caucasian) & $200(82.6 \%)$ & $106(82.2 \%)$ & 0.09 & $218(89.3 \%)$ & $123(93.9 \%)$ & 0.14 & $227(93.4 \%)$ & $128(93.4 \%)$ & 1.00 \\
\hline Body Mass Index $\left(\mathrm{kg} / \mathrm{m}^{2}\right)$ & $29.1 \pm 5.09$ & $29.4 \pm 5.42$ & 0.65 & $30.0 \pm 5.70$ & $29.9 \pm 6.22$ & 0.95 & $28.8 \pm 5.50$ & $28.7 \pm 5.71$ & 0.81 \\
\hline Death & $21(8.7 \%)$ & $9(7.0 \%)$ & 0.57 & $32(13.1 \%)$ & $17(13.0 \%)$ & 0.97 & $57(23.5 \%)$ & $18(13.1 \%)$ & 0.02 \\
\hline Family history of CAD & $101(41.7 \%)$ & $63(48.8 \%)$ & 0.19 & $79(32.4 \%)$ & $45(34.4 \%)$ & 0.7 & $89(36.6 \%)$ & $41(29.9 \%)$ & 0.19 \\
\hline Smoking & $149(62.9 \%)$ & $65(50.4 \%)$ & 0.02 & $141(59.7 \%)$ & $72(55.4 \%)$ & 0.42 & $134(55.6 \%)$ & 69 (50.4\%) & 0.33 \\
\hline Hypertension & $172(71.1 \%)$ & $98(76.6 \%)$ & 0.26 & $180(74.1 \%)$ & $93(71.0 \%)$ & 0.52 & $181(74.5 \%)$ & $113(83.1 \%)$ & 0.05 \\
\hline Diabetes mellitus & $94(38.8 \%)$ & $33(25.6 \%)$ & 0.01 & $73(29.9 \%)$ & $33(25.2 \%)$ & 0.33 & $96(39.5 \%)$ & 47 (34.3\%) & 0.32 \\
\hline Peripheral arterial disease & $29(12.0 \%)$ & $13(10.1 \%)$ & 0.58 & $36(14.8 \%)$ & $7(5.3 \%)$ & 0.006 & $39(16.0 \%)$ & $14(10.2 \%)$ & 0.12 \\
\hline Renal failure on dialysis & $3(1.2 \%)$ & $1(0.8 \%)$ & 0.68 & $3(1.2 \%)$ & $5(3.8 \%)$ & 0.1 & $8(3.3 \%)$ & $9(6.6 \%)$ & 0.14 \\
\hline Myocardial infarction & $134(55.4 \%)$ & $51(39.5 \%)$ & 0.004 & $132(54.1 \%)$ & $52(39.7 \%)$ & 0.008 & $130(53.5 \%)$ & $38(27.7 \%)$ & $<0.001$ \\
\hline History of $\mathrm{PCl}$ & $71(29.3 \%)$ & $23(17.8 \%)$ & 0.02 & $66(27.0 \%)$ & $31(23.7 \%)$ & 0.48 & $58(23.9 \%)$ & $23(16.8 \%)$ & 0.11 \\
\hline Prior CABG & $7(2.9 \%)$ & $2(1.6 \%)$ & 0.51 & $7(2.9 \%)$ & $1(0.8 \%)$ & 0.27 & $9(3.7 \%)$ & $2(1.5 \%)$ & 0.34 \\
\hline Prior heart failure & $37(15.3 \%)$ & $7(5.4 \%)$ & 0.005 & $41(16.8 \%)$ & $9(6.9 \%)$ & 0.007 & $45(18.5 \%)$ & $11(8.0 \%)$ & 0.01 \\
\hline Prior cerebrovascular event & $31(12.8 \%)$ & $8(6.2 \%)$ & 0.048 & $39(16.0 \%)$ & $11(8.4 \%)$ & 0.04 & $50(20.6 \%)$ & $12(8.8 \%)$ & 0.003 \\
\hline COPD & $30(12.4 \%)$ & $10(7.9 \%)$ & 0.18 & $34(13.9 \%)$ & $16(12.2 \%)$ & 0.64 & $34(14.0 \%)$ & $16(11.7 \%)$ & 0.52 \\
\hline \multicolumn{10}{|l|}{ Preoperative CCS } \\
\hline none & $0(0 \%)$ & $1(0.8 \%)$ & 0.14 & $2(0.8 \%)$ & $0(0 \%)$ & 0.02 & $2(0.8 \%)$ & $0(0 \%)$ & $<0.001$ \\
\hline Class I & $42(17.6 \%)$ & $24(18.6 \%)$ & & $34(13.9 \%)$ & $32(24.4 \%)$ & & $41(17.2 \%)$ & $28(20.6 \%)$ & \\
\hline Class II & $61(25.5 \%)$ & $45(34.9 \%)$ & & $74(30.3 \%)$ & $47(35.9 \%)$ & & $46(19.3 \%)$ & 49 (36.0\%) & \\
\hline Class III & $68(28.5 \%)$ & $33(25.6 \%)$ & & $75(30.7 \%)$ & $29(22.1 \%)$ & & $83(34.9 \%)$ & $45(33.1 \%)$ & \\
\hline Class IV & $68(28.5 \%)$ & $26(20.2 \%)$ & & $59(24.2 \%)$ & $23(17.6 \%)$ & & $66(27.7 \%)$ & $14(10.3 \%)$ & \\
\hline \multicolumn{10}{|l|}{ NYHA } \\
\hline Class I & $22(17.9 \%)$ & $21(19.4 \%)$ & 0.21 & $25(29.2 \%)$ & $25(24.0 \%)$ & 0.31 & $30(23.8 \%)$ & $22(21.2 \%)$ & 0.03 \\
\hline Class II & $33(26.8 \%)$ & $40(37.0 \%)$ & & 42 (32.3\%) & $39(37.5 \%)$ & & $26(20.6 \%)$ & 33 (31.7\%) & \\
\hline Class III & 44 (35.8\%) & $26(24.1 \%)$ & & 37 (28.5\%) & 19 (18.3\%) & & 38 (30.2\%) & 37 (35.6\%) & \\
\hline Class IV & $24(19.5 \%)$ & $21(19.4 \%)$ & & $26(20.0 \%)$ & 21 (20.2\%) & & 32 (25.4\%) & 12 (11.5\%) & \\
\hline Ejection Fraction (\%) & $39.8 \pm 12.1$ & $41.0 \pm 10.8$ & 0.32 & $39.1 \pm 12.1$ & $41.6 \pm 10.4$ & 0.04 & $39.3 \pm 12.8$ & $41.2 \pm 12.4$ & 0.17 \\
\hline
\end{tabular}


Table 1 Baseline characteristics (demographic and presentation) of coronary artery bypass patients according to neutrophil lymphocyte ratios (NLR) and surgical approach (MICS vs. Sternotomy CABG) (Continued)

\begin{tabular}{|c|c|c|c|c|c|c|c|c|c|}
\hline \multicolumn{10}{|c|}{ Preoperative laboratory values } \\
\hline Leukocyte (k/cc) & $7.42 \pm 2.31$ & $7.20 \pm 2.03$ & 0.37 & $8.09 \pm 2.22$ & $7.61 \pm 2.20$ & 0.05 & $9.70 \pm 3.13$ & $8.87 \pm 2.71$ & 0.01 \\
\hline Neutrophils (k/cc) & $4.06 \pm 1.35$ & $3.94 \pm 1.29$ & 0.40 & $5.28 \pm 1.55$ & $4.97 \pm 1.51$ & 0.06 & $7.45 \pm 2.82$ & $6.76 \pm 2.45$ & 0.01 \\
\hline Lymphocytes (k/cc) & $2.45 \pm 1.10$ & $2.40 \pm 0.763$ & 0.40 & $1.91 \pm 0.566$ & $1.79 \pm 0.533$ & 0.06 & $1.35 \pm 0.462$ & $1.29 \pm 0.455$ & 0.24 \\
\hline Monocytes (k/cc) & $0.62 \pm 0.286$ & $0.62 \pm 0.241$ & 0.80 & $0.65 \pm 0.263$ & $0.60 \pm 0.225$ & 0.12 & $0.69 \pm 0.299$ & $0.62 \pm 0.237$ & 0.02 \\
\hline Creatinine $(\mathrm{mg} / \mathrm{dl})$ & $1.06 \pm 0.741$ & $1.07 \pm 0.983$ & 0.99 & $1.14 \pm 0.711$ & $1.19 \pm 1.02$ & 0.56 & $1.26 \pm 0.61$ & $1.18 \pm 0.604$ & 0.06 \\
\hline Glucose (mg/dl) & $124.0 \pm 48.9$ & $122.4 \pm 52.4$ & 0.76 & $126.1 \pm 49.3$ & $110.8 \pm 41.6$ & 0.002 & $136.2 \pm 58.8$ & $121.5 \pm 46.3$ & 0.01 \\
\hline
\end{tabular}

CABG coronary artery bypass grafting, CAD coronary artery disease, CCS Canadian Cardiovascular Society Angina classification system, COPD chronic obstructive pulmonary disease, NYHA New York Heart Association Functional Classification, $\mathrm{PCl}$ percutaneous coronary intervention. Categorical variables were presented as frequencies and percentages; continuous variables were presented as means and standard deviations. 
Table 2 Operative and post-operative characteristics (management and laboratory) of coronary artery bypass patients according to neutrophil lymphocyte ratios (NLR) and surgical approach (MICS vs. Sternotomy CABG)

\begin{tabular}{|c|c|c|c|c|c|c|c|c|c|}
\hline \multirow[t]{2}{*}{ Variables } & \multicolumn{2}{|c|}{ NLR $<2.3(\mathrm{~N}=371)$} & \multirow[t]{2}{*}{$p$ Value } & \multicolumn{2}{|c|}{$2.3 \leq \mathrm{NLR} \leq 3.4(\mathrm{~N}=375)$} & \multirow[t]{2}{*}{$p$ Value } & \multicolumn{2}{|c|}{ NLR $>3.4(\mathrm{~N}=380)$} & \multirow[t]{2}{*}{$p$ Valu } \\
\hline & Sternotomy CABG & MICS & & Sternotomy CABG & MICS & & Sternotomy CABG & MICS & \\
\hline $\mathrm{n}$ & 242 & 129 & & 245 & 130 & & 243 & 137 & \\
\hline \multicolumn{10}{|l|}{ In-hospital medication } \\
\hline Aspirin & $238(98.3 \%)$ & $128(99.2 \%)$ & 0.66 & $234(95.9 \%)$ & $130(100 \%)$ & 0.02 & 237 (97.5\%) & $137(100 \%)$ & 0.09 \\
\hline Clopidogrel & $110(45.5 \%)$ & $111(86.0 \%)$ & $<0.001$ & $128(52.5 \%)$ & $115(88.5 \%)$ & $<0.001$ & $120(49.4 \%)$ & $116(84.7 \%)$ & $<0.001$ \\
\hline Beta-blockers & $232(95.9 \%)$ & $124(96.1 \%)$ & 0.91 & $230(94.3 \%)$ & $128(98.5 \%)$ & 0.06 & $231(95.1 \%)$ & 135 (98.5\%) & 0.08 \\
\hline Angiotensin convertase inhibitor & $84(34.7 \%)$ & $44(34.1 \%)$ & 0.91 & $96(39.3 \%)$ & $31(23.8 \%)$ & 0.00 & $104(42.8 \%)$ & $45(32.8 \%)$ & 0.06 \\
\hline Angiotensin receptor blocker & $29(12.0 \%)$ & $13(10.1 \%)$ & 0.58 & $17(7.0 \%)$ & $13(10.0 \%)$ & 0.30 & $19(7.8 \%)$ & $17(12.4 \%)$ & 0.14 \\
\hline Warfarin & $18(7.4 \%)$ & $7(5.4 \%)$ & 0.46 & $18(7.4 \%)$ & $8(6.2 \%)$ & 0.66 & $26(10.7 \%)$ & $9(6.6 \%)$ & 0.18 \\
\hline Statin & $232(95.9 \%)$ & $127(98.4 \%)$ & 0.23 & $229(93.9 \%)$ & $126(96.9 \%)$ & 0.20 & $228(93.8 \%)$ & $132(96.4 \%)$ & 0.29 \\
\hline \multicolumn{10}{|l|}{ Medication on discharge } \\
\hline Aspirin & $237(98.3 \%)$ & $125(97.7 \%)$ & 0.70 & $234(95.9 \%)$ & 127 (97.7\%) & 0.56 & $230(95.4 \%)$ & $131(97.8 \%)$ & 0.26 \\
\hline Clopidogrel & $66(27.4 \%)$ & $101(78.9 \%)$ & $<0.001$ & $83(34.0 \%)$ & $105(80.8 \%)$ & $<0.001$ & $64(26.6 \%)$ & $104(77.6 \%)$ & $<0.001$ \\
\hline Beta-blockers & $212(88.0 \%)$ & $114(89.1 \%)$ & 0.76 & $216(88.5 \%)$ & 117 (90.0\%) & 0.66 & $208(86.3 \%)$ & $123(91.8 \%)$ & 0.11 \\
\hline Angiotensin convertase inhibitor & $70(29.0 \%)$ & $32(25.0 \%)$ & 0.41 & $67(27.5 \%)$ & $24(18.5 \%)$ & 0.05 & $75(31.1 \%)$ & $27(20.1 \%)$ & 0.02 \\
\hline Angiotensin receptor blocker & $1(0.4 \%)$ & $4(3.1 \%)$ & 0.05 & $4(1.7 \%)$ & $1(0.8 \%)$ & 0.66 & $2(0.8 \%)$ & $7(5.2 \%)$ & 0.01 \\
\hline Warfarin & $30(12.4 \%)$ & $17(13.3 \%)$ & 0.82 & $27(11.1 \%)$ & $12(9.2 \%)$ & 0.58 & $43(17.8 \%)$ & $11(8.2 \%)$ & 0.01 \\
\hline Statin & $220(91.3 \%)$ & 119 (93.0\%) & 0.57 & $222(91.0 \%)$ & $120(92.3 \%)$ & 0.66 & $218(90.5 \%)$ & $123(91.8 \%)$ & 0.67 \\
\hline \multicolumn{10}{|l|}{ Operative data } \\
\hline Internal mammary artery utilized & $231(95.5 \%)$ & $127(98.4 \%)$ & 0.23 & $230(94.3 \%)$ & $128(97.7 \%)$ & 0.13 & $221(90.9 \%)$ & $134(97.8 \%)$ & 0.01 \\
\hline Number of conduits used for bypass & $3.28 \pm 0.991$ & $2.12 \pm 0.673$ & $<0.001$ & $3.26 \pm 0.886$ & $2.28 \pm 0.806$ & $<0.001$ & $3.12 \pm 0.910$ & $2.17 \pm 0.753$ & $<0.001$ \\
\hline Cardiopulmonary bypass & $27(11.2 \%)$ & $6(4.7 \%)$ & 0.04 & $30(12.3 \%)$ & $7(5.3 \%)$ & 0.03 & $18(7.4 \%)$ & $8(5.8 \%)$ & 0.56 \\
\hline \multicolumn{10}{|l|}{ Postoperative complications } \\
\hline Renal Failure on Dialysis & $1(0.4 \%)$ & $2(1.6 \%)$ & 0.28 & $3(1.2 \%)$ & $4(3.1 \%)$ & 0.25 & $12(4.9 \%)$ & $3(2.2 \%)$ & 0.19 \\
\hline New onset atrial fibrillation & $33(13.6 \%)$ & $22(17.1 \%)$ & 0.38 & $40(16.4 \%)$ & $30(22.9 \%)$ & 0.12 & $48(19.8 \%)$ & $43(31.4 \%)$ & 0.01 \\
\hline Septicemia & $0(0 \%)$ & $5(3.9 \%)$ & 0.005 & $0(0 \%)$ & $2(1.5 \%)$ & 0.12 & $6(2.5 \%)$ & $2(1.5 \%)$ & 0.72 \\
\hline
\end{tabular}

Categorical variables were presented as frequencies and percentages; continuous variables were presented as means and standard deviations. CABG coronary artery bypass grafting. 


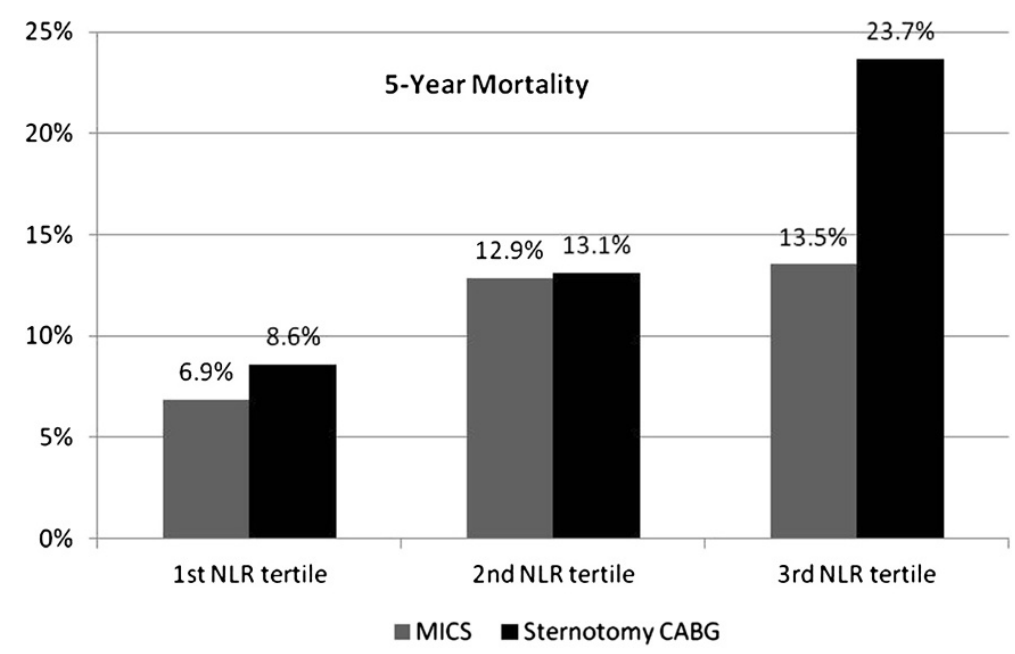

Figure 1 The 5-year all-cause mortality after coronary bypass surgery according to preoperative neutrophil lymphocyte ratio (NLR) and surgical approach(MICS vs. Sternotomy CABG). According to Fisher's exact test the 5-year mortality rates were higher in the sternotomy CABG patients with NLR $>3.4$ compared to MICS with NLR $>3.4$ ( $p$ value $<0.0001$ ). MICS = minimal invasive cardiac surgery; $C A B G=$ coronary artery bypass grafting. The average follow-up was $49 \pm 15.2$ months (range 21-70 months). Total of 467 patients had 5-year follow up period.

Fisher's exact test) (Figure 1). Similarly, Kaplan-Meier curves showed significant difference (Log rank [MantelCox] $\mathrm{p}<0.0001)$ mortality in the highest NLR tertile (NLR $>3.4$ ) compared to the middle (NLR 2.3-3.4) and the lowest (NLR <2.3) NLR tertiles (Figure 2). Table 3 demonstrates the univariate screening analysis (the individual Cox regressions examining the association between each variable and overall survival). In our study population, the variables associated with increased mortality were age, chronic pulmonary disease, congestive heart failure, prior cerebrovascular event, diabetes, family history of coronary artery disease, pre- and post-operative renal failure requiring dialysis, intraoperative use of cardiopulmonary bypass (CPB), elevated preoperative neutrophil, NLR, serum creatinine,

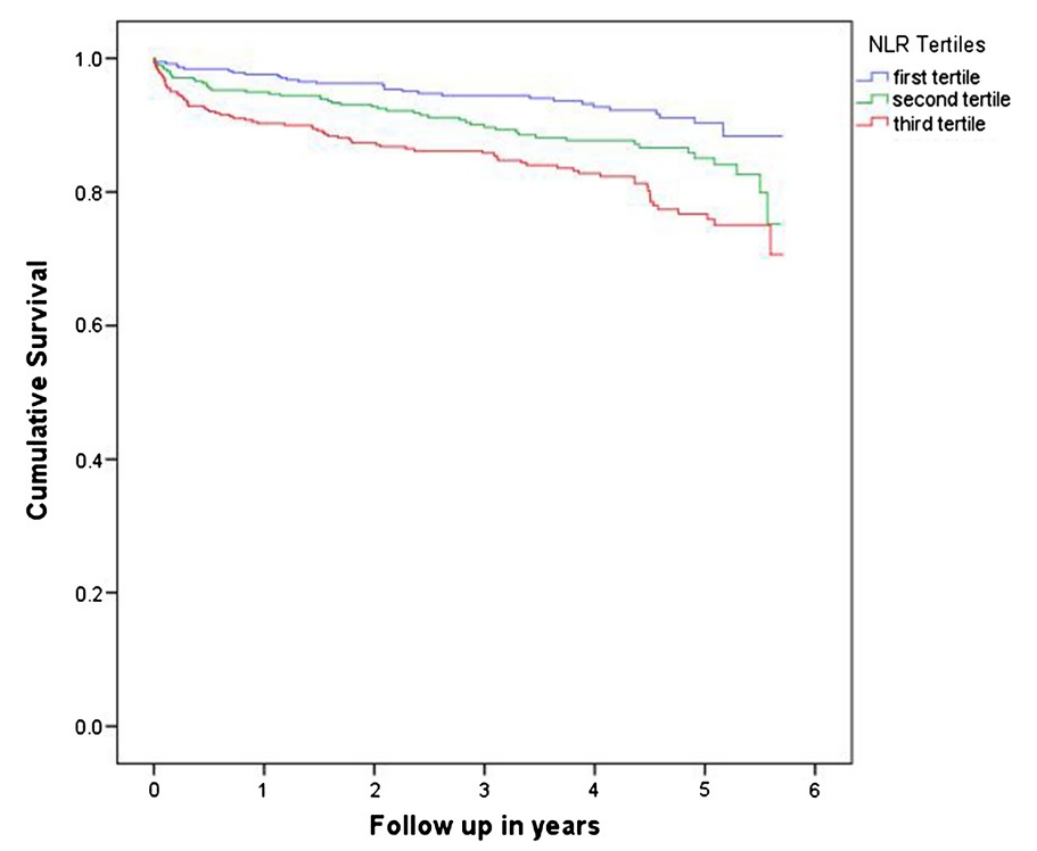

Figure 2 Kaplan-Meier Curve of mortality among patients undergoing coronary artery bypass surgery according to their neutrophil lymphocyte ratio (NLR) tertiles. Log rank (Mantel-Cox) $p<0.0001$. The average follow-up was $49 \pm 15.2$ months (range 21-70 months). Total of 467 patients had 5-year follow up period. 
Table 3 Hazard ratios of baseline characteristics for allcause mortality among coronary artery bypass surgery patients (univariate analysis)

\begin{tabular}{|c|c|c|}
\hline Variable & $\begin{array}{l}\text { Hazard ratio ( } 95 \% \\
\text { confidence interval) }\end{array}$ & $p$ Value \\
\hline Male & $0.63(0.45-0.87)$ & 0.005 \\
\hline Age (per year) & $1.06(1.06-1.09)$ & $<0.001$ \\
\hline Race (Caucasian) & $1.40(0.78-2.53)$ & 0.26 \\
\hline \multicolumn{3}{|l|}{$\begin{array}{l}\text { Body Mass Index } \\
\left(\mathrm{kg} / \mathrm{m}^{2}\right)(31+)\end{array}$} \\
\hline $18-25$ & $1.35(0.90-2.03)$ & 0.14 \\
\hline $26-30$ & $1.11(0.75-1.64)$ & 0.60 \\
\hline Ejection fraction (\%) & $1.01(0.99-1.02)$ & 0.46 \\
\hline Hypertension & $1.31(0.89-1.93)$ & 0.17 \\
\hline Smoking & $0.89(0.65-1.23)$ & 0.48 \\
\hline Chronic pulmonary disease & $2.02(1.37-2.96)$ & $<0.001$ \\
\hline Prior myocardial infarction & $1.00(0.73-1.37)$ & 0.99 \\
\hline Prior coronary angioplasty & $1.16(0.81-1.65)$ & 0.41 \\
\hline $\begin{array}{l}\text { Prior coronary artery bypass } \\
\text { graft }\end{array}$ & $0.67(0.21-2.09)$ & 0.48 \\
\hline Congestive heart failure & $1.64(1.11-2.43)$ & 0.01 \\
\hline Prior cerebrovascular event & $1.79(1.22-2.62)$ & 0.003 \\
\hline $\begin{array}{l}\text { Family history of coronary } \\
\text { artery disease }\end{array}$ & $0.56(0.39-0.80)$ & 0.001 \\
\hline Renal Failure on Dialysis & $3.95(2.19-7.12)$ & $<0.001$ \\
\hline Diabetes Mellitus & $1.60(1.17-2.21)$ & 0.004 \\
\hline Peripheral arterial disease & $1.39(0.91-2.14)$ & 0.12 \\
\hline Pre-Operative CCS & $1.01(0.87-1.18)$ & 0.85 \\
\hline Pre-Operative NYHA & $0.86(0.71-1.06)$ & 0.15 \\
\hline Pre-Operative Leukocyte & $1.05(0.99-1.11)$ & 0.11 \\
\hline Pre-Operative Neutrophils & $1.08(1.02-1.15)$ & 0.01 \\
\hline Pre-Operative Lymphocytes & $0.69(0.55-0.88)$ & 0.003 \\
\hline Pre-Operative Monocytes & $1.46(0.91-2.36)$ & 0.11 \\
\hline Pre-Operative Creatinine & $1.20(1.09-1.31)$ & $<0.001$ \\
\hline Pre-Operative Glucose & $1.00(1.00-1.01)$ & $<0.001$ \\
\hline Pre-Operative NLR & $1.09(1.06-1.13)$ & $<0.001$ \\
\hline \multicolumn{3}{|l|}{$\begin{array}{l}\text { Operative and } \\
\text { postoperative data }\end{array}$} \\
\hline $\begin{array}{l}\text { Internal mammary artery } \\
\text { used }\end{array}$ & $0.35(0.21-0.57)$ & $<0.001$ \\
\hline $\begin{array}{l}\text { Number of conduits used } \\
\text { for bypass }\end{array}$ & $0.96(0.82-1.13)$ & 0.64 \\
\hline Renal failure on dialysis & $6.80(3.91-11.80)$ & $<0.001$ \\
\hline Cardiopulmonary bypass & $1.76(1.11-2.79)$ & 0.01 \\
\hline New onset atrial fibrillation & $2.03(1.41-2.91)$ & $<0.001$ \\
\hline Septicemia & $6.95(3.54-13.65)$ & $<0.001$ \\
\hline
\end{tabular}

CCS Canadian Cardiovascular Society Angina classification system, NLR neutrophil lymphocyte ratio, NYHA New York Heart Association Functional Classification. serum glucose, postoperative septicemia, and new onset atrial fibrillation (Table 3). Male gender, use of internal mammary artery and higher lymphocyte count were associated with lower mortality. Elevated WBC, neutrophil, NLR and lower lymphocyte count were significant predictors of mortality in the univariate screening models. In the multivariate Cox proportional hazard models, including the confounding variables, NLR was the only independent predictor of long-term mortality (HR per unit increase in NLR was 1.06 , CI 95\% was 1.014-1.099, $\mathrm{p}=0.008$ ) (Table 4).

When patients were divided into two groups according to the median NLR $(\mathrm{NLR}=3)$ of the whole population, we found 624 patients with NLR $<3$ and 502 with NLR $\geq 3$. Among the patients with NLR $<3$, there was no significant difference in the overall survival between the MICS and sternotomy CABG $(21 / 221=9.5 \%$ vs. $40 / 403=9.9 \%, p=1)$. Contrarily, the patients with $\mathrm{NLR} \geq 3$ who underwent MICS had a significantly lower mortality compared to sternotomy CABG $(23 / 176=13.1 \%$ vs. $70 / 326=21.5 \%$, $\mathrm{p}=0.022$ ). Kaplan-Meier curves showed significant difference in (Log rank [Mantel-Cox] $\mathrm{p}<0.002$ ) mortality between the MICS and sternotomy CABG with NLR $\geq 3$ compared to NLR $<3, \mathrm{p}=0.002$ (Figure 3). Additionally, the patients with NLR $\geq 3$ who underwent MICS had a significantly lower mortality $(20 / 166=12 \%$ vs. $63 / 293=21.5 \%$, $\mathrm{p}=0.011)$ compared to those who underwent off-pump sternotomy CABG, after excluding MICS patients who underwent $\mathrm{CPB}$. Among the patients with NLR $\geq 3$, multivariate regression models demonstrated that MICS CABG decreased the odd ratios of all-cause mortality by $56 \%$ and $50 \%$ according to model A and B, respectively (Table 5), compared to sternotomy.

\begin{tabular}{|c|c|c|c|}
\hline Variables & HR & $95 \% \mathrm{Cl}$ & $p$ Value \\
\hline Pre-Operative NLR (per unit) & 1.06 & $1.01-1.10$ & 0.008 \\
\hline Age (per year) & 1.07 & $1.05-1.09$ & $<0.001$ \\
\hline Male gender & 0.73 & $0.51-1.03$ & 0.06 \\
\hline Family history of coronary artery disease & 0.71 & $0.49-1.04$ & 0.07 \\
\hline Smoking & 1.37 & $0.96-1.95$ & 0.08 \\
\hline Preoperative renal failure on dialysis & 5.56 & $2.98-10.4$ & $<0.001$ \\
\hline Chronic pulmonary disease & 1.73 & $1.15-2.59$ & 0.008 \\
\hline In-hospital angiotensin convertase inhibitor & 1.41 & $1.00-1.99$ & 0.05 \\
\hline In-hospital statin & 0.37 & $0.20-0.67$ & 0.001 \\
\hline Aspirin on discharge & 0.29 & $0.15-0.56$ & $<0.001$ \\
\hline Postoperative renal failure on dialysis & 2.34 & $1.16-4.71$ & 0.01 \\
\hline Postoperative septicemia & 2.99 & $1.29-6.93$ & 0.01 \\
\hline Preoperative Glucose (pre mg/dl) & 1.01 & $1.00-1.01$ & $<0.001$ \\
\hline
\end{tabular}

$N L R$ neutrophil lymphocyte ratio, $H R$ hazard ratio, $\mathrm{Cl}$ confidence interval. 

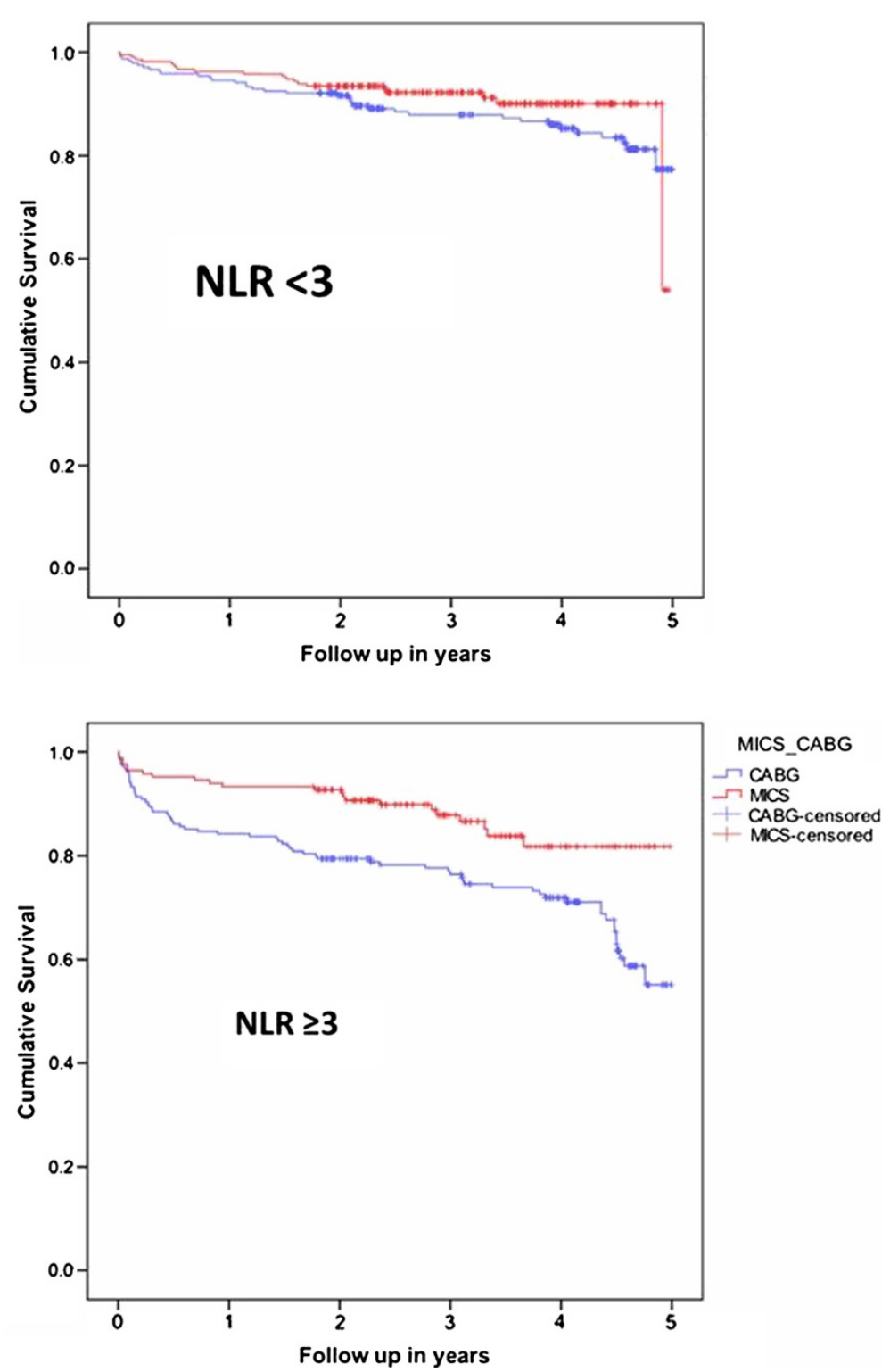

Figure 3 Kaplan-Meier Curve of mortality among patients undergoing coronary bypass surgery according to their neutrophil lymphocyte ratio (NLR) cutoffs: NLR $<3$ and NLR $\geq \mathbf{3}(\mathbf{p}=\mathbf{0 . 0 0 2}$ ). The average follow-up was $49 \pm 15.2$ months (range 21-70 months). Total of 467 patients had 5-year follow up period.

\section{Discussion}

The principle finding of our study is that elevated preoperative NLR independently predicts long term mortality in patients undergoing coronary artery bypass surgery via minimally invasive and sternotomy approaches. Those with elevated preoperative NLR $\geq 3$ had better survival with MICS than with sternotomy CABG after adjusting for confounding variables. Elevated NLR was associated with a higher prevalence of diabetes, renal failure, and elevated serum creatinine and glucose. This association concurs with existing literature that describes these conditions as proinflammatory [6,7].
Similar to a prior study, we have observed an association between elevated preoperative NLR and the development of postoperative new onset atrial fibrillation [4].

Similar to prior studies, we found that NLR is superior to WBC, neutrophil and lymphocyte counts in predicting mortality. Because it is a ratio, NLR is relatively more stable than individual leukocytic parameters that are easily altered by many simple conditions (e.g. dehydration, over-hydration, diluted blood specimens, in-vitro blood specimen handling). In the NLR, the numerator is the neutrophil count (a higher neutrophil count is associated with an increased risk of death) and 
Table 5 Multivariate logistic regression models for allcause mortality among patients with preoperative neutrophil lymphocyte ratio $\geq 3$

\begin{tabular}{|c|c|c|c|}
\hline Variable & $\begin{array}{l}\text { Odd } \\
\text { ratio }\end{array}$ & $\begin{array}{l}\text { 95\% confidence } \\
\text { interval }\end{array}$ & $p$ Value \\
\hline \multicolumn{4}{|l|}{ Model A } \\
\hline $\begin{array}{l}\text { MICS vs. Sternotomy } \\
\text { CABG }\end{array}$ & 0.44 & $0.24-0.78$ & 0.005 \\
\hline $\begin{array}{l}\text { Internal mammary artery } \\
\text { used }\end{array}$ & 0.39 & $0.17-0.89$ & 0.02 \\
\hline $\begin{array}{l}\text { Postoperative new onset } \\
\text { atrial fibrillation }\end{array}$ & 1.72 & $1.01-2.93$ & 0.04 \\
\hline $\begin{array}{l}\text { Beta blockers on } \\
\text { discharge }\end{array}$ & 0.57 & $0.30-1.09$ & 0.09 \\
\hline In-hospital clopidogrel & 1.56 & $0.92-2.63$ & 0.09 \\
\hline \multicolumn{4}{|l|}{ Model B } \\
\hline $\begin{array}{l}\text { MICS vs. Sternotomy } \\
\text { CABG }\end{array}$ & 0.50 & $0.28-0.90$ & 0.02 \\
\hline Age (per year) & 1.08 & $1.05-1.11$ & $<0.001$ \\
\hline $\begin{array}{l}\text { Preoperative renal failure } \\
\text { on dialysis }\end{array}$ & 10.05 & $3.46-29.19$ & $<0.001$ \\
\hline $\begin{array}{l}\text { Internal mammary artery } \\
\text { used }\end{array}$ & 0.48 & $0.20-1.12$ & 0.08 \\
\hline
\end{tabular}

Model A included history of prior coronary angioplasty, MICS vs. sternotomy $C A B G$, postoperative new onset atrial fibrillation, use of internal mammary artery, use of cardiopulmonary bypass, clopidogrel on discharge and inhospital, coumadin on discharge, beta blockers on discharge.

Model B included age, gender, family history of coronary artery disease, history of renal dialysis, statin on discharge, coumadin on discharge, use of internal mammary artery, MICS vs. sternotomy CABG and postoperative new onset atrial fibrillation.

MICS minimal invasive cardiac surgery (thoracotomy), CABG coronary artery bypass graft.

the denominator is the lymphocyte (a lower lymphocyte count is associated with an increased risk of death). This makes the NLR an augmented predictor of mortality. We hypothesize that an elevated neutrophil count is a marker for an active inflammatory process and a low lymphocyte count is a marker of failure to control this active inflammatory process. Many studies have demonstrated that neutrophils play a major role in atherosclerosis and plaque instability through various mediators such as neutrophil gelatinase-associated lipocalin (NGAL), neutrophil-elastase, myeloperoxidase, defensins and cathelicidin [8-11]. Studies have also demonstrated that preoperative statin therapy increases neutrophil apoptosis and reduces neutrophil-endothelial adhesion after cardiac surgery. These desirable statin effects were irrespective of lipid lowering action $[12,13]$.

We hypothesize that a reduced peripheral blood lymphocyte count acts as a marker for an ongoing nonspecific atherosclerotic inflammatory process. Lymphopenia has been shown to be associated with atherosclerosis progression and major adverse cardiac outcomes $[14,15]$. Lymphocytes modulate smooth muscle proliferation via interferon gamma [16] which controls the course of luminal narrowing in atherosclerosis.

Prior studies evaluated $\mathrm{CPB}$ as a confounding variable to the inflammatory response after MICS versus sternotomy CABG. Gu et al. demonstrated in a randomized controlled trial (31 patients in each arm) that anterolateral thorocotomy was associated with a lower inflammatory response (leukocyte elastase, platelet $\beta$ thromboglobulin, and complement $C$ ) than sternotomy CABG [17]. However some of the sternotomy CABG patients in this study underwent $\mathrm{CPB}$, which is believed by many to contribute greatly to the postoperative inflammatory response. According to our multivariate analysis, NLR predicted mortality after adjusting for CPB. Similarly in patients with NLR >3, MICS had better survival than sternotomy CABG after adjusting for CPB. Moreover, among patients with NLR $>3$, MICS off-pump had a significant lower mortality compared to off-pump sternotomy CABG. These results demonstrate that $\mathrm{CPB}$ was not a confounding variable to the superiority of MICS in these patients with elevated inflammatory marker. However, our study included only 96 on-pump CABG patients, which requires further studies with larger number of on-pump patients.

We compared the inflammatory response between MICS and sternotomy CABG. Gu et al. prospectively studied the inflammatory response in patients that underwent $\mathrm{CABG}$ without $\mathrm{CPB}$. They found the inflammatory response in patients without $\mathrm{CPB}$ was lower in the anterolateral thoracotomy group compared to the sternotomy group [18]. In a randomized controlled trial of 30 patients, Diegeler et al. studied various inflammatory markers in three groups of CABG: sternotomy CABG with $\mathrm{CPB}$, off-pump sternotomy CABG and limited anterior thoracotomy CABG approaches. The anterior thoracotomy approach had a lower interleukin 8 (pro-inflammatory marker) and a higher interleukin 10 (anti-inflammatory marker) than the off-pump sternotomy CABG [19]. The complement 3d, TNF receptor p55 and p75 showed a significant increase in both on and off-pump sternotomy CABG, but no change from the anterior thoracotomy group [19]. In a study of patients that underwent lung volume reduction surgery, bilateral thoracoscopic surgery had reduced inflammatory cytokines compared to sternotomy [20]. Likewise, in an experimental study using animals that underwent CPB with different surgical approaches, avoidance of full-sternotomy was associated with a reduced level of cytokines and inflammatory response. The animals without sternotomy had the lowest pro-inflammatory (IL-6 and IL-8) cytokines compared to mini and full sternotomy [21].

The superiority of MICS to sternotomy CABG in the group with high NLR may be related to thymus 
dysfunction after sternotomy. In a case-control pediatric study, $29 \%$ of children with sternotomy after cardiac surgery had an identifiable thymus on 14-month magnetic resonance image compared to $92 \%$ in the control group who had no cardiac surgery. The authors suggested that postoperative residual thymic tissue does not regenerate [22]. Additionally, in a study that included pediatric and adult populations, cardiothoracic surgery and incidental thymectomy were associated with immune dysregulation and unclear factors that altered the regulatory $\mathrm{T}$ cells [23]. In adult patients, thymectomies were followed by immune dysregulation and development of autoimmune diseases [24,25].

\section{Limitations}

This is a single center study with patients who were retrospectively enrolled from our database. We have not included the operating surgeon in our data, which could be a possible confounding variable. The MICS patients with high NLR had a higher rate of internal mammary artery use compared to the sternotomy group with high NLR. Although the internal mammary artery use was included in the multivariate analysis, randomized prospective studies are needed to alleviate such significant difference in operative variables. We used all-cause mortality rather than cardiac related mortality. We also did not obtain data regarding other major cardiovascular adverse events during the follow-up period (e.g. myocardial infarction, stroke and later need for revascularization). There is the possibility of missing a number of primary outcomes by using the social security death index and medical records to assess the survival status. The smoking data are missing exact duration of smoking or cessation. Another limitation to our study is the lack of measurement for known inflammatory markers such as interleukins, C-reactive protein and erythrocyte sedimentation rate. Moreover, we had mostly off-pump CABG patients (91\%), which limits the generalizability of our findings.

\section{Conclusion}

Elevated preoperative NLR is an independent predictor of long-term mortality after CABG. Patients with preoperative NLR $\geq 3$ had a better 5 -year overall survival in MICS versus sternotomy CABG. This effect was not attributed to cardiopulmonary bypass or any other confounding variables. Further studies are needed to explain the mechanism and evaluate therapeutic applications to these findings.

\section{Abbreviations}

NLR: Neutrophil lymphocyte ratio; CABG: Coronary artery bypass grafting surgery; MICS: Minimally invasive coronary artery bypass surgery.

\section{Competing interests}

The authors declare that they have no competing interests.

\section{Authors' contributions}

BA researched data, contributed to the discussion, and wrote the manuscript. JTM designed the protocol, contributed to the discussion, and reviewed/edited the manuscript. MAS collected, researched data, contributed to the discussion, and reviewed/edited the manuscript. RB analyzed and researched data. JPN contributed to the discussion and reviewed/edited the manuscript. All authors read and approved the final manuscript.

\section{Acknowledgments}

We acknowledge the cardiothoracic surgery team: Physician Assistants, Nursing and Clinical staff at Staten Island University Hospital; the Physician volunteer staff who assisted in review of electronic medical records: Emad Barsoum MD, Ravi Kavuda MD, Gerges Makar MD, and Fahad Bin Nayim MD; other volunteers who assisted with data collection: Ankit Christie, Korrine Compton, James Kenny, Silivia Khalil, Yan Pertsov, David Pitera, Natasha Povar, Jason Selim, and Elias Youssef; and Ricardo Crosby in extracting the data points from the Centricity DMS STS database. Special thanks to Dr. Neeraj Shah for assistance in reviewing the final submission.

\section{Disclosures}

No potential conflicts of interest relevant to this article.

\section{Oral presentation}

International Congress of Coronary Artery Disease (ICCAD), 2011 Venice, Italy.

\section{Author details}

${ }^{1}$ Department of Surgery, Staten Island University Hospital, 475 Seaview Ave, Staten Island, NY, USA. ${ }^{2}$ Cardiothoracic Surgery Department, Staten Island University Hospital, 475 Seaview Ave, Staten Island, NY, USA.

Received: 17 April 2013 Accepted: 12 September 2013 Published: 27 September 2013

\section{References}

1. Núñez J, Núñez E, Bodí V, Sanchis J, Miñana G, Mainar L, Santas E, Merlos P, Rumiz E, Darmofal H, Heatta AM, Llàcer A: Usefulness of the neutrophil to lymphocyte ratio in predicting long-term mortality in ST segment elevation MI. Am J Cardiol 2008, 101:747-752.

2. Papa A, Emdin M, Passino C, Michelassi C, Battaglia D, Cocci F: Predictive value of elevated neutrophil-lymphocyte ratio on cardiac mortality in patients with stable CAD. Clin Chim Acta 2008, 395:27-31.

3. Azab B, Zaher M, Weiserbs KF, Torbey E, Lacossiere K, Gaddam S, Gobunsuy R, Jadonath S, Baldari D, McCord D, Lafferty J: Usefulness of neutrophil to lymphocyte ratio in predicting short- and long-term mortality after nonST-elevation MI. Am J Cardiol 2010, 106(4):470-476.

4. Gibson PH, Croal BL, Cuthbertson BH, Small GR, Ifezulike Al, Gibson G, Jeffrey RR, Buchan KG, El-Shafei H, Hillis GS: Preoperative NLR and outcome from CABG. Am Heart J 2007, 154:995-1002.

5. McGinn JT Jr, Usman S, Lapierre H, Pothula VR, Mesana TG, Ruel M: Minimally invasive coronary artery bypass grafting: dual-center experience in 450 consecutive patients. Circulation 2009, 120(11 Suppl):S78-S84

6. Dandona P, Aljada A, Bandyopadhyay A: Inflammation: the link between insulin resistance, obesity and diabetes. Trends Immunol 2004, 25(1):4-7.

7. Miyamoto T, Carrero JJ, Stenvinkel P: Inflammation as a risk factor and target for therapy in CKD. Curr Opin Nephrol Hypertens 2011, 20(6):662-668.

8. Hemdahl AL, Gabrielsen A, Zhu C, Eriksson P, Hedin U, Kastrup J, Thorén P, Hansson GK: Expression of neutrophil gelatinase- associated lipocalin in atherosclerosis and MI. Arterioscler, Thromb, Vasc Biol 2006, 26(1):136-142.

9. Henriksen PA, Sallenave JM: Human neutrophil elastase: mediator and therapeutic target in atherosclerosis. Int J Biochem Cell Biol 2008, 40(6-7):1095-1100.

10. Roman RM, Camargo PV, Borges FK, Rossini AP, Polanczyk CA: Prognostic value of myeloperoxidase in coronary artery disease: comparison of unstable and stable angina patients. Coron Artery Dis 2010, 21(3):129-136.

11. Döring Y, Drechsler M, Wantha S, Kemmerich K, Lievens D, Vijayan S, Gallo RL, Weber C, Soehnlein O: Lack of neutrophil-Derived CRAMP reduces atherosclerosis in mice. Circ Res 2012, 110(8):1052-1056. 
12. Chello M, Anselmi A, Spadaccio C, Patti G, Goffredo C, Di Sciascio G, Covino E: Simvastatin increases neutrophil apoptosis and reduces inflammatory reaction after coronary surgery. Ann Thorac Surg 2007, 83(4):1374-1380.

13. Chello M, Mastroroberto P, Patti G, D'Ambrosio A, Morichetti MC, Di Sciascio G, Covino E: Simvastatin attenuates leucocyte-endothelial interactions after coronary revascularisation with CPB. Heart 2003, 89(5):538-543.

14. Major AS, Fazio S, Linton MF: B-lymphocyte deficiency increases atherosclerosis in LDL receptor-null mice. Arterioscler, Thromb, Vasc Biol 2002, 22(11):1892-1898.

15. Núñez J, Sanchis J, Bodí V, Núñez E, Mainar L, Heatta AM, Husser O, Miñana G, Merlos P, Darmofal H, Pellicer M, Llàcer A: Relationship between low lymphocyte count and major cardiac events in patients with acute chest pain, a non-diagnostic electrocardiogram and normal troponin levels. Atherosclerosis 2009, 206(1):251-257.

16. Hansson GK, Holm J, Holm S, Fotev Z, Hedrich HJ, Fingerle J: Tlymphocytes inhibit the vascular response to injury. Proc Natl Acad Sci USA 1991, 88:10530-10534.

17. Gu YJ, Mariani MA, van Oeveren W, Grandjean JG, Boonstra PW: Reduction of the inflammatory response in patients undergoing minimally invasive CABG. Ann Thorac Surg 1998, 65(2):420-424.

18. Gu YJ, Mariani MA, Boonstra PW, Grandjean JG, van Oeveren W: Complement activation in coronary artery bypass grafting patients without cardiopulmonary bypass: the role of tissue injury by surgical incision. Chest 1999, 116(4):892-898.

19. Diegeler A, Doll N, Rauch T, Haberer D, Walther T, Falk V, Gummert J, Autschbach R, Mohr FW: Humoral immune response during CABG: A comparison of limited approach, "off-pump" technique, and conventional CPB. Circulation 2000, 102(19 Suppl 3):III95-III100.

20. Friscia ME, Zhu J, Kolff JW, Chen Z, Kaiser LR, Deutschman CS, Shrager JB: Cytokine response is lower after lung volume reduction through bilateral thoracoscopy versus sternotomy. Ann Thorac Surg 2007, 83(1):252-256.

21. Hayashi Y, Sawa Y, Nishimura M, Satoh H, Ohtake S, Matsuda H: Avoidance of full sternotomy: effect on inflammatory cytokine production during CPB in rats. J Card Surg 2003, 18(5):390-395.

22. MacDonald K, Mackenzie S: What happens to the thymus in children who have undergone a median sternotomy? Pediatr Radiol 2009, 39(6):616-621.

23. Halnon NJ, Cooper P, Chen DY, Boechat MI, Uittenbogaart CH: Immune dysregulation after cardiothoracic surgery and incidental thymectomy: maintenance of regulatory $T$ cells despite impaired thymopoiesis. Clin Dev Immunol 2011, 2011:915864.

24. Mevorach D, Perrot S, Buchanan NM, Khamashta M, Laoussadi S, Hughes GR, Menkes CJ: Appearance of systemic lupus erythematosus after thymectomy: four case reports and review of the literature. Lupus 1995, 4(1):33-37.

25. Gerli R, Paganelli R, Cossarizza A, Muscat C, Piccolo G, Barbieri D, Mariotti S, Monti D, Bistoni O, Raiola E, Venanzi FM, Bertotto A, Franceschi C: Longterm immunologic effects of thymectomy in patients with myasthenia gravis. J Allergy Clin Immunol 1999, 103(5 Pt 1):865-872.

doi:10.1186/1749-8090-8-193

Cite this article as: Azab et al:: Elevated preoperative neutrophil/ lymphocyte ratio as a predictor of increased long-term survival in minimal invasive coronary artery bypass surgery compared to sternotomy. Journal of Cardiothoracic Surgery 2013 8:193.

\section{Submit your next manuscript to BioMed Central and take full advantage of:}

- Convenient online submission

- Thorough peer review

- No space constraints or color figure charges

- Immediate publication on acceptance

- Inclusion in PubMed, CAS, Scopus and Google Scholar

- Research which is freely available for redistribution
C Biomed Central 